\title{
ASPERGILOSE INVASIVA DO SEIO ESFENOIDAL E PARALISIA DO SEXTO NERVO
}

MARIA INES DE VILHENA LANA-PEIXOTO * MARCO AURELIO LANA-PEIXOTO*

RESUMO - A aspergilose do seio esfenoidal é doença rara e pode se apresentar sob diferentes formas clínicas devido a envolvimento de diversas estruturas anatomicamente adjacentes ao seio esfenoidal. Relatamos o caso de uma paciente com 74 anos de idade, diabetica, com paralisia do sexto nervo esquerdo secundária a aspergilose do seio esfenoidal. Nåo havla história de cefaléia ou de queixas sugestivas de alergia respiratória. A tomografia computadorizada revelou lesão etmoídeo-esfenoidal à esquerda, com presença de imagem cálcica em seu interior e destruição óssea. A paciente foi submetida a cirurgia com retirada de material necrótico e debridamento da lesão, seguida de tratamento com anfotericina B e 5-fluorocitosina. Exame histológico revelou a presenca de hifas sugestivas de Aspergillus sp. Após três meses de tratamento a paciente apresentou recuperação total da paresia do nervo abducente. $O$ diagnóstico clínico pré-operatório de aspergilose do seio esfenoldal é difficil. No entanto, a presença de imagem cálcica ou de densidade metálica à radiografia simples de crânio ou à tomografia computadorizada sugere fortemente o diagnóstico. O exame hihstológico revela a presença de hifas dicotomatosas em 45,0 típicas do Aspergillus. O tratamento inclui excisão e debridamento da lesão seguida do uso de anfotericina B associada a 5-fluorocitosina ou rifampicina.

PALAVRAS CHAVE: aspergilose, seio esfenoidal, VI nervo craniano.

Invasive aspergillosis of the sphenoid sinus manifested as an isolated sixth nerve palsy.

SUMMARY - A case of aspergillosis of the sphenoid sinus manifested as an isolated sixth nerve palsy occurred in a 74-year old diabetic woman who had no complaints of headache or symptoms suggestive of sinusitis. A CT scan demonstrated a large mass occupying the sphenoid and ethmoid sinuses extending posteriorly to the clivus. There was a calcific density within the opacified sinus and bony erosion of the sphenoid walls and the sella turcica. The patient underwent a sublabial transseptal sphenoidotomy with removal of necrotic material and debridment of the surrounding tissue. Histologic examination revealed granulation tissue with chronic inflammatory cells and abundant dichotomously branching hyphae. Postoperatively the patient was given amphotericin B and 5-fluorocytosine. Three months later the sixth nerve palsy had completely cleared and the patient had no other complaint. Sphenoid sinus aspergillosis is a rare disease and may have variable clinical manifestations according to involvement of different structures located closely to the sinus. Our patient developed an isolated sixth nerve palsy which was at onset considered to be caused by diabetes. Computerized tomography scans disclosed abnormalities strongly indicative of invasive aspergillosis. It illustrates the need of appropriate work-up in cases of an isolated sixth nerve palsy even in patients with diabetes or other risk factors.

KEY WORDS: aspergillosis, sphenoid sinus, VI cranial nerve.

A aspergilose, causada por uma ou mais espécies de Aspergillus é afecção incomum, afetando principalmente os brônquios, pulmões e o canal auditivo externo mas, ocasionalmente, pode envolver a pele, unhas, seios paranasais, órbita e meninges. A aspergilose dos seios paranasais até recentemente era considerada doença rara, mas seu diagnóstico tem aumentado provavelmente devido ao melhor reconhecimento

* Professor Adjunto de Neurologia, Faculdade de Medicina, Universidade Federal de Minas Gerais (UFMG).

Dra. Maria Ines de Vilhena Lana-Peixoto - Departamento de Neurologia, Faculdade de Medicina, UFMG - Av. Alfredo Balena 190 - 30130 Belo Horizonte MG - Brasil. 
da entidade pelos médicos, à idade mais avançada da população e ao aumento do número de pacientes imunodeprimidos 10,24,31. Embora rara, a aspergilose é a infecção fúngica mais comum dos seios paranasais. O envolvimento do seio esfenoidal carrega prognóstico pior, enquanto o do seio maxilar é mais benigno 19. A aspergilose do seio esfenoidal tem sido descrita associada a trombose do seio carótido-cavernoso 23 , a oftalmoplegia dolorosa com paralisia do nervo oculomotor 2,14 e simulando tumor hipofisário 5 .

Relatamos aqui um caso de paralisia isolada do nervo abducente esquerdo como forma de apresentação da aspergilose invasiva do sistem nervoso central, em paciente diabética, a partir de infecção do seio esfenoidal, enfatizando a necessidade de propedêutica adequada mesmo em casos de paralisias oculomotoras isoladas em pacientes com outros fatores de risco.

\section{OBSERVACXAO}

HLR, paciente do sexo feminino, faiodérmica, com 74 anos de idade, veio referida ao nosso Departamento com queixa de diplopia horizontal de três meses de duração atribuída a diabetes. Havia história de diabetes mellitus, não insulina-dependente há 8 anos. A paciente não referia a cefaléia ou queixas sugestivas de sinusite ou de alergia respiratória. o exame neurológico revelou estrabismo convergente com paralisia do músculo reto lateral esquerdo (Fig. 1). Exame dos demais nervos cranianos não demonstrou outras alterações.
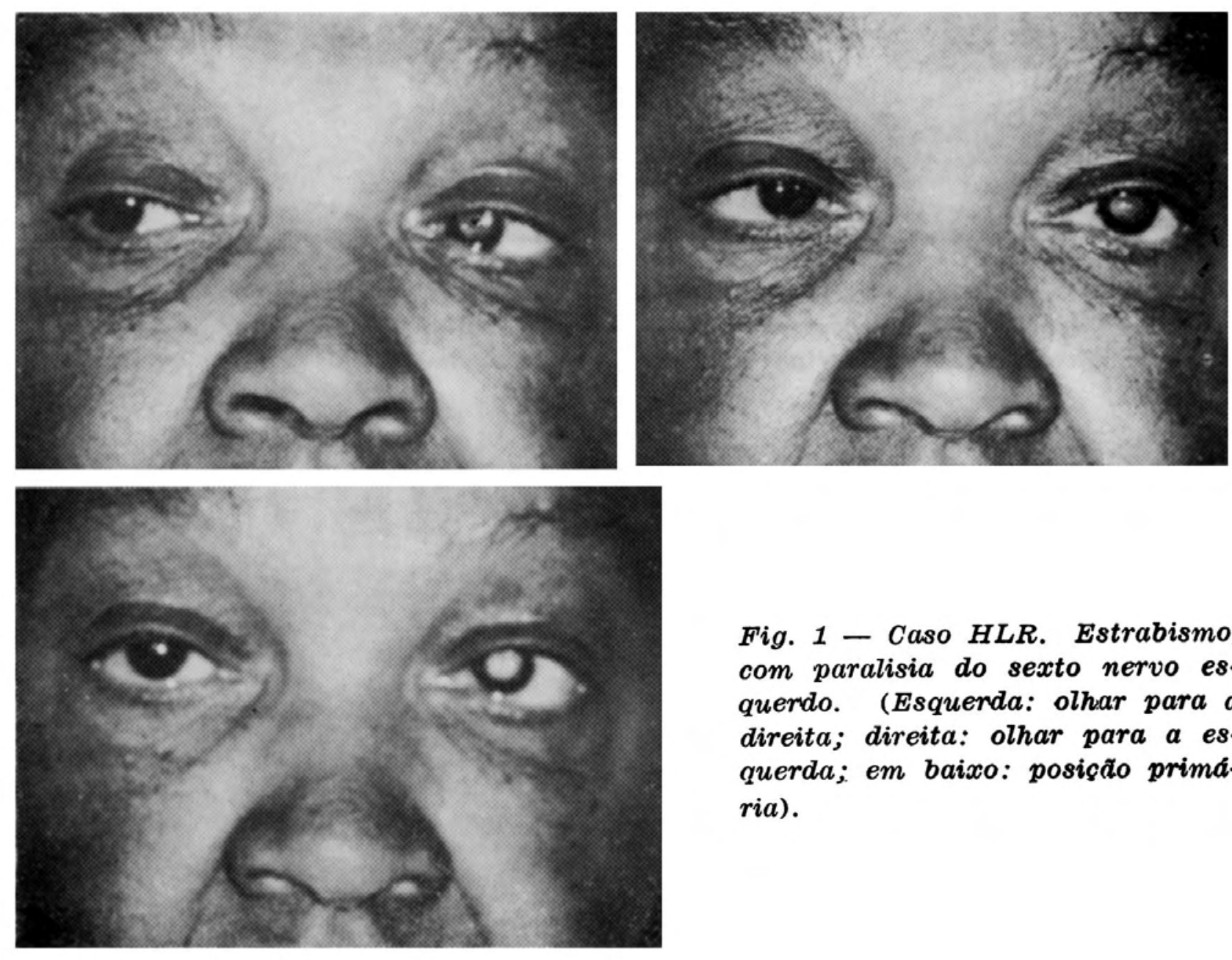

Fig. 1 - Caso HLR. Estrabismo com paralisia do sexto nervo esquerdo. (Esquerda: olhar para a direita; direita: olhar para a esquerda; em baixo: posiça primária).

Havia hipoestesia e hipoalgesia em luvas e meias nos 4 membros, os reflexos osteotendíneos estavam diminuídos distalmente e havia dismetria discreta bilateral a prova dedo-nariz. A pressão arterial era $130 / 90 \mathrm{mmHg}$. O hemograma não revelou alterações e a velocidade de hemossedimentação foi $12 \mathrm{~mm}$ na $1^{\text {a }}$ hora. A glicemia de jejum foi $168 \mathrm{mg} \%$ enquanto as taxas de colesterol, triglicérides, uréia e creatinina se encontravam em níveis normais. Serologia para lues foi negativa. A tomografia computadorizada de crânio mostrou lesăo isodensa etmoídeo-esfenoidal esquerda com presença de imagem cálcica em seu interior e destruição óssea do assoalho do seio esfenoidal, da borda lateral e do dorso da sela túrcica, estendendo-se ao clivus (Fig. 2). A paciente foi submetida a cirurgia por via transeptal, com retirada de material gelatinoso esbranquiçado e debridamento de todo o seio esfenoidal sob visão microscópica. Exame histológico deste material revelou abundante tecido necrótico permeado por raras trabéculas ósseas, com a presença do numerosas hifas dicotomatosas 
em 45', morfologicamente sugestivas de Aspergillus sp. junto a discreto infiltrado inflamatório focal com predomínio de células mononucleares e áreas de hemorragia recente (Fig. 3). A paciente foi tratada com anfotericina $B$ e 5-fluorocitosina, apresentando após três meses recuperação total da paralisia do nervo abducente.
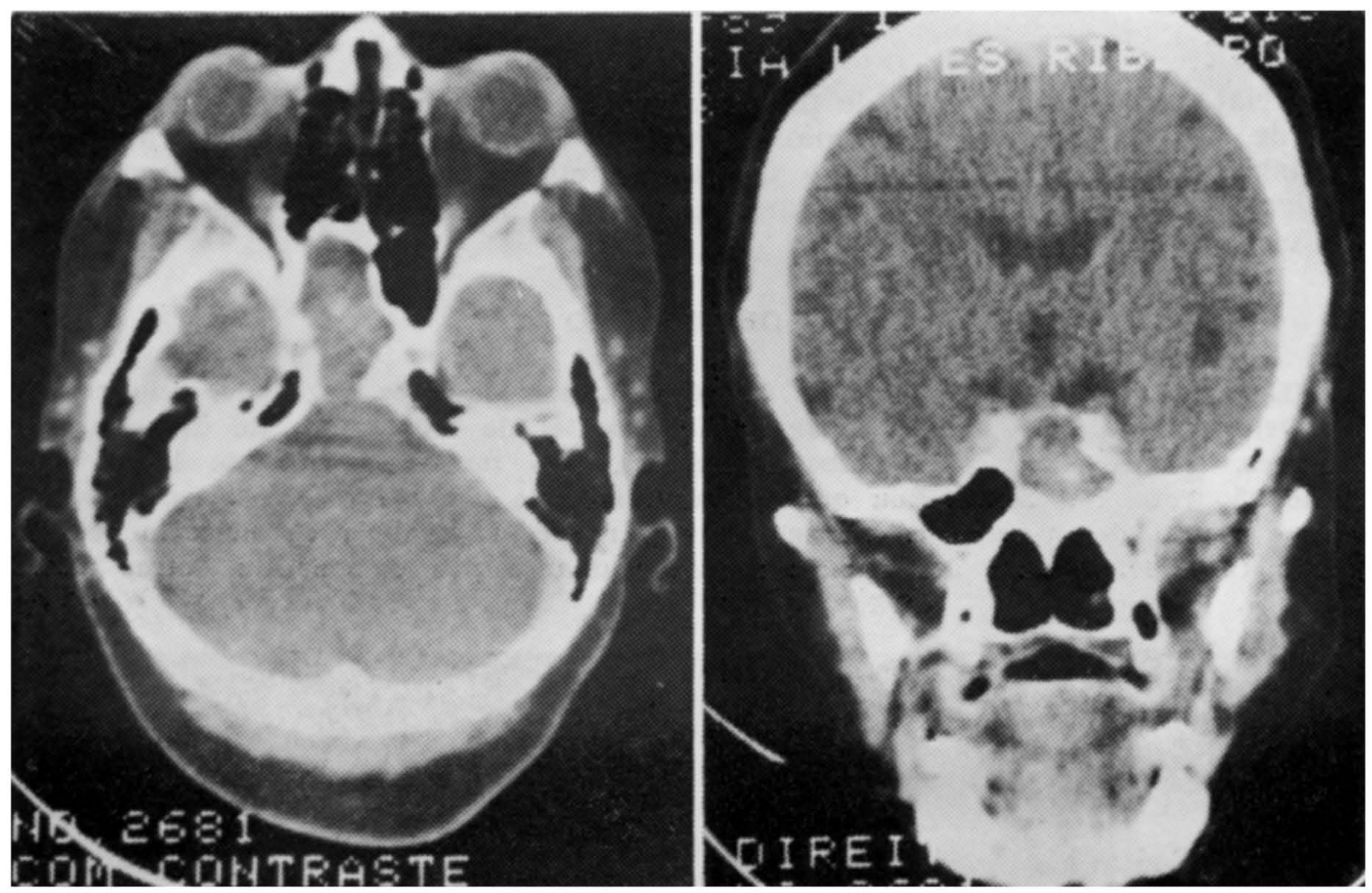

Fig. \& - Caso HLR. Tomografia computadorizada de cranio demonstrando grande massa etmoideo-esfenoidal, com destruição da sela túrcica e presença de imagem de densidade calcica em seu interior (esquerda: corte transversal; direita: corte coronal).

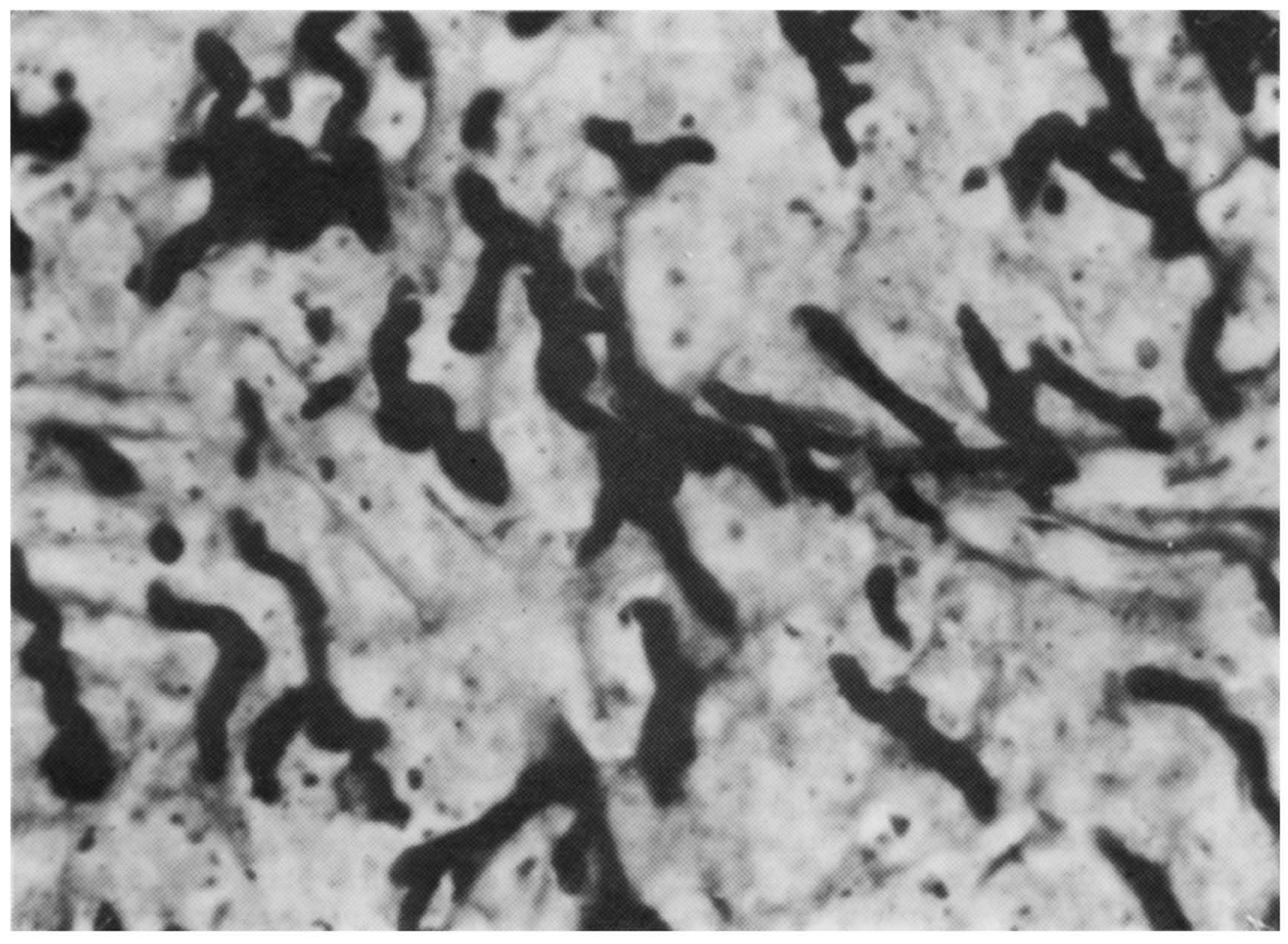

Fig. 3 - Caso HLR. Microscopia da lesião mostrando hifias de Aspergillus com típicas ramificaç̃es dicotomatosas em $45^{\circ}$ (Coloracão pela prata, $\times 400$ ). 


\section{COMENTÁRIOS}

O Aspergillus é fungo da classe Ascomycetas de distribuição generalizada em todo o mundo, isolado de várias fontes como o solo, folhas, material em decomposição, poeira, grãos e mofos de alimento. Seus esporos penetram no sistema respiratório do homem onde, sob condições especiais, o fungo pode tornar-se patogênico.

Embora o homem entre em contato com grande variedade de espécies de Aspergillus, apenas 7 espécies são implicadas em infecções humanas. Aspergillus fumigatus é responsável por cerca de $90 \%$ destas infecções 20 , seguido por Aspergillus flavus. O clima quente e úmido, com sua elevada tendência de ocasionar rinosinusite alérgica, vasomotora e infecciosa, alterando as condições fisiológicas das vias aéreas superiores predispõe a infecção fúngica. Vários autores sugerem que o Aspergillus torna-se patogênico apenas quando os seios paranasais se encontram relativamente anaeróbicos 1,21. Desvios do septo nasal e a presença de pólipos são também fatores predisponentes à colonização dos seios paranasais pelo Aspergillus. A aspergilose dos seios paránasais é ainda assim rara, exceto no Sudão onde a doença é endêmica 21,27. Os seios maxilares e etmoidais são mais comumente afetados enquanto o envolvimento dos seios esfenoidais traz o risco de extensão da infecção ao sistema nervoso, principalmente em pacientes diabéticos, debilitados ou imunodeprimidos. A infecção dos seios paranasais pode ocorrer em quatro formas distintas - 1. Forma não-invasiva, em geral no hospedeiro imunologicamente não comprometido, podendo envolver um ou mais seios paranasais, não havendo acometimento da mucosa nem erosão óssea; a cura é alcançada por remoção do conteúdo do seio infectado, incluindo a sua mucosa, e restabelecimento da aeração $3,18,22$. 2. Forma invasiva, acometendo também pessoas sadias, caracterizada por envolvimento da mucosa do seio e por destruição óssea, podendo haver extensão da infecção para o interior da órbita e do crânio, tornando difícil o diagnóstico diferencial com neoplasias 12,24,30; mesmo com tratamento agressivo, com debridamento cirúrgico da lesão e uso de drogas antimicóticas sistêmicas, a taxa de mortalidade pode chegar a $75 \% 10$. 3. Forma fulminante, ocorre em imunodeprimidos com neutropenia, usualmente secundária ao uso de drogas imunossupressoras para o tratamento de neoplasias; há formação de lesão crostosa escura, não-dolorosa no septo nasal com rápida progressão da necrose atingindo os tecidos adjacentes; em série de 1331 pacientes recebendo tratamento quimioterápico para neoplasias hematológicas a aspergilose dos seios paranasais ocorreu em $20 \%$ dos pacientes 28; a mortalidade é alta mesmo quando agentes antifúgicos e a intervenção cirúrgica são instituídos; quando há invasão orbitária ou craniana o curso é em geral fatal 10,28. 4. Forma alérgica recorrente, assemelha-se à aspergilose alérgica broncopulmonar, com achados histológicos de eosinófilos mucinosos, cristais de Charcot-Leyden, debris celulares e ocasionais hifas fúngicas; o tratamento inclui corticosteróides sistêmicos, antibióticos para controle das infeccões secundárias e tratamento da alergia; o prognóstico é excelente, embora recorrências sejam comuns 11,13.

$O$ diagnóstico de infecção fúngica dos seios deve ser suspeitado quando uma «sinusite» não responde a terapia com antibióticos ou irrigações, quando o processo está confinado a um único seio ou quando há evidência de destruição óssea. $O$ diagnóstico diferencial nestes pacientes inclui neoplasias, que devem ser afastadas por biópsia 20. Quando o seio esfenoidal é acometido, o diagnóstico clínico é ainda mais difícil em razão do possível envolvimento das várias estruturas anatômicas - nervos óptico e abducente, dura-máter, hipófise, seio cavernoso, artéria carótida, artéria esfenopalatina, nervo, gânglio e canal esfenopalatinos - intimamente ligadas a ele 8 . O mais comum dos sintomas é cefaleia, em geral de longa duração e de localização variável, como retro-orbitária, frontal, parieto-occipital ou no vértex. Sintomas convencionais de sinusite, em geral não são proeminentes 30 . Envolvimento orbitário com proptose unilateral, dor retro-orbitária ou temporal, diplopia e diminuição da acuidade visual pode ocorrer simulando lesão primária da órbita ou massa intracraniana. O Aspergillus frequentemente ocasiona arterite com resultante trombose, oclusão ou aneurisma, especialmente das artérias carótida interna e cerebral média. Neste aspecto ele se assemelha à mucormicose que tem acentuada predileção pela invasão de vasos 15 . Casos de infarto hemorrágico intracerebral têm sido relatados, presumivelmente devido a trombose ou ruptura de vasos envolvidos por angiite $\mathbf{9 , 2 6}$. Trombose do seio cavernoso 23 e abscesso hipofisário $5,7,30$ têm sido relatados.

A radiografia simples de crânio e a tomografia computadorizada podem ser úteis no diagnóstico. Desordens que ocasionam opacificação dos seios paranasais incluem sinusites bacterianas agudas e crônicas, mucoceles e tumores malígnos. 
Stammberger e col.24 relataram em 59 casos de aspergilose dos seios paranasais a presença de imagem cálcica, às vezes de densidade metálica, no centro da área opacificada em 27 pacientes $(45,8 \%)$. Estes autores afirmam que na ausência de história clínica de corpo estranho, o aparecimento de imagem cálcica ou de densidade metálica nos seios paranasais é altamente sugestiva de aspergilose. Recentemente Horton e Osguthorpe 11 descreveram imagens de calcificação no interior do seio esfenoidal opacificado à tomografia computadorizada em dois pacientes com aspergilose, possibilitando o diagnóstico pré-operatório. Imagem por ressonância magnética foi descrita em um caso de aspergilose do seio esfenoidal com síndrome do seio cavernoso 6. Os achados de área de baixa intensidade, representando a massa fúngica, em área de alta intensidade em $T_{1}$ e $T_{2}$ podem ser característicos 6.

Exames laboratoriais incluem testes de imunodifusão, cultura da cavidade nasal e indentificação microscópica do fungo por biópsia da lesão. Os testes de imunodifusão podem ser específicos para aspergiloma paranasal mas não são disponíveis em todos os centros 16. Culturas da cavidade nasal não tem mostrado grande utilidade. Apenas $58 \%$ dos pacientes com cultura positiva para Aspergillus, numa série de 1331 pacientes com cultura da cavidade nasal, apresentavam aspergilose dos seios paranasais, enquanto os outros $42 \%$ apresentavam sinusite por outras causas 28 .

O exame histológico permanece como o meio mais fácil e eficiente para o diagnóstico da aspergilose dos seios paranasais. A presença de pequenas hifas septadas dicomatosas com ramificações em $45^{\circ}$ é diagnóstica. Há necrose fibrinóide do tecido colágeno adjacente e formação de pseudotubérculos semelhantes aos encontrados em reações de hipersensibilidade prolongada, infiltração de linfócitos e células plasmáticas ao longo dos vasos sanguíneos nos tecidos adjacentes e o desenvolvimento de arterite 23. Achados operatórios de granulações acinzentadas ou marrom-escuras sugerem infecção por Aspergillus 12,19.

A aspergilose paranasal pode responder a tratamento cirúrgico conservador que restabelece a aeração do seio envolvido 29 . No entanto, em casos de aspergilose invasiva ou envolvimento de seio esfenoidal há indicação de cirurgia com remoção de todo o material necrótico e debridamento do tecido adjacente em conjunção ao uso de agentes antifúngicos. A combinação de anfotericina B e 5-fluorocitosina ou rifampicina apresenta efeitos aditivos ou sinergísticos 30 , embora algumas espécies de Aspergillus possam ser resistentes a essas drogas 4 . Como o oxigênio em elevadas concentrações é fungicida, o uso de oxigênio hiperbárico pode ser útil na complementação do tratamento 25 .

O presente relato tem interesse particular devido à ocorrência de paralisia isolada do sexto nervo, inicialmente atribuída ao diabetes, ilustrando então a importância do uso dos métodos de imagem no diagnóstico diferencial das etiologias destas paralisias, mesmo em casos em que fatores de risco bem conhecidos estejam presentes.

\section{REFERENCIAS}

1. Adams VF. Infection involving the ethmoid, maxillary and sphenoid sinuses and the orbit due to Aspergillus fumigatus. Arch Surg 1933, 26:999-1009.

2. Ardouin M, Urvoy $M$, Bourdiniere J, Le Clech G, Viallee B, Scarabin JM. Aspergillose pseudo-tumorale du sinus shénoïdal (ou l'etiologie d'une paralysie unilatérale du III). J Fr Ophtalmol 1979, 2:625-627.

3. Axelson H. Carlsoo B, Weibring J, Winblad B. Aspergillosis of the maxillary sinus. Acta Otolaryngol 1978, 86:303-308.

4. Bennett JE. Chemotherapy of systemic micosis (First of two parts). N Engl J Med 1974, $290: 30-32$.

5. Fuchs HA, Evans RM, Gregg CR. Invasive aspergillosis of the sphenoid sinus manifested as a pituitary tumor. Southern Med J 1985, 78:1365-1367.

6. Fujiwara S, Fujino $H$, Nogami $K$, Nishio S, Fukui $M$. Aspergillosis of the sphenoid sinus with cavernous sinus syndrome. Neuroradiology 1989, 31:443-446.

7. Goldhammer $Y$, Smith JF Yates RM. Mycotic intrasellar abscess. Trans Am Ophthalmol Soc 1974, 72:65-78.

8. Goldstein MF, Atlkins PC, Cogen FC, Kornstein MJ, Levine RS, Zweirman B. Allergic aspergillus sinusitis: J Allergy Clin Immuno 1985, 76:515-524.

9. Greevic, Matthews WF. Pathologic changes in acute disseminated aspergillosis: particular involvement of the central nervous system. Am J Clin Pathol 1959, 32:536-551. 
10. Holt GR, Standefer JA, Brown WE, Gates GA. Infectious diseases of the sphenoid sinus. Laryngoscope 1984, $94: 330-335$.

11. Horton WD, Osguthorpe DJ. CT findings in sphenoid sinus aspergillosis. Otolaringol Head Neck Surg 1989, 100:606-609.

12. Jahrsdoerfer RA, Ejercits VS, Johns MME, Cantrell RM, Sydnor JB. Aspergillosis of the nose and paranasil sinus. Am $J$ Otolaryngol 1979, 1:6-14.

13. Katzenstein A, Sale SR, Greenberger PA. Allergic aspergillus sinusitis. J Allergy Clin Immunol 1983, 72:89-93.

14. Lemoine JJ, Le Rebeller MJ, Bagot D'Arc M. Ophtalmoplegie amaurotique a bascule associée a une aspergillose du sinus sphenoidal. Bull Soc Ophtalmol Fr 1984, 84:787-790.

15. Lowe JT, Hudson WR. Rhinocerebral phycomycoses and internal carotid artery thrombosis. Arch Otolaryngol 1975, 101:100-103.

16. Mahgoub ELS. Mycological and serological studies on aspergillus flavus isolated from paranasal aspergilloma in Sudan. J Trop Med Hyg 1971, 74:162-165.

17. McGill TJ, Simpson G, Healy GB. Fulminant aspergillosis of the nose and paranasal sinuses: a new clinical entity. Laryngoscope 1980, 90:748-754.

18. McGuirt WF, Harril JA. Paranasal sinus aspergillosis. Laryngoscope 1979, 89:1563-1568.

19. Merkle D. Yarington CT, Winterbauer RH. Aspergillosis of the maxillary sinuses in otherwise healthy patients. Laryngoscope 1985, 95:776-779.

30. Miglets AW. Saunders WH, Leona A. Aspergillosis of the sphenoid sinus. Arch Otolaryngol 1978, 104:47-50.

21. Milosev B, Mahgoub ELs, Abdel Aal AO, El Hanan MA. Primary aspergilloma of the paranasal sinuses in the Sudan. Br $J$ Surg 1969, 56:132-137.

22. Rommett JL, Newman RK. Aspergillosis of the nose and paranasal sinuses. Laryngoscope 1982, 92:764-766.

23. Sekhar LN, Dujovny M, Rao GR. Carotid-cavernous sinus thrombosis caused by Aspergillus fumigatus: case report. J Neurosurg 1980, 52:120-125.

24. Stammberger $H$, Jakse $R$, Beaufort $F$. Aspergillosis of the paranasal sinuses: $X$-ray diagnosis, histopathology and clinical aspects.' Ann Otol Rhinol Laryngol 1984, 93: 251-256.

25. Stevens MH. Primary fungal infections of the paranasal sinuses. Am J Otolaryngol 1981, $2: 348-351$.

26. Tveten L. Cerebral mycosis: a clinico-pathological report of four cases. Acta Neurol Scand 1965, 41:19-33.

27. Veress B. Malik OA, El Tayeb AA, El Daoud S, Mahgoub ES, El Hassan AM. Further observations on the primary paranasal aspergillus granuloma in the Sudan: a morphological study of 46 cases. Am J Trop Med Hyg 1973, 22:765-772.

28. Viollier AF, Peterson DE. DeJongh CA, Newman KA, Gray WG, Sutherland JC, Moody MA, Schimpff SC. Aspergillus sinusitis in cancer patients. Cancer 1986, 58:366-371.

24. Wiarder FR, Chickes PG, Hudson WR. Aspergillosis of the paranasal sinuses. Arch Otolaryngol 1975, 101:683-685.

3i. Yu VL, Wagner GE, Shadomy S. Sino-orbital aspergillosis treated with combination antifungal therapy. JAMA 1980, 244:814-815

31. Yumots E, Kitani S, Okamura H, Yanagihara N. Sino-orbital aspergillosis associated with total ophthalmoplegia. Laryngoscope 1985, 95:190-192. 